\title{
Prospective investigation of change in the prostate-specific antigens after various urologic procedures
}

This article was published in the following Dove Press journal:

Clinical Interventions in Aging

29 July 2015

Number of times this article has been viewed

\author{
Seung Chol Park, 'Yu Seob \\ Shin, ${ }^{2}$ Li Tao Zhang, ${ }^{2}$ Dal Sik \\ $\mathrm{Kim},{ }^{3}$ Sung Zoo Kim, ${ }^{4} \mathrm{Nam}$ \\ Cheol Park, ${ }^{5}$ Tai Young Ahn, ${ }^{6}$ \\ Je Jong Kim, ${ }^{7}$ Sung Won \\ Lee, ${ }^{8}$ Insuk So, ${ }^{9}$ Jong Kwan \\ Park $2,10,11$
}

'Department of Urology, Wonkwang University School of Medicine and Hospital, Iksan, South Korea; ${ }^{2}$ Department of Urology, ${ }^{3}$ Department of Laboratory Medicine, ${ }^{4}$ Department of Physiology, Medical School, Institute for Medical Sciences, Chonbuk National University, Jeonju, ${ }^{5}$ Department of Urology, College of Medicine, Pusan National University Hospital, Busan, ${ }^{6}$ Department of Urology, College of Medicine, Ulsan University, Seoul, ${ }^{7}$ Department of Urology, College of Medicine, Korea University, Seoul, ${ }^{8}$ Department of College of Medicine, SungKyunkwan University, Seoul, ${ }^{9}$ Department of Physiology, College of Medicine, Seoul National University, Seoul, ${ }^{10}$ Research Institute of Clinical Medicine, Chonbuk National University, and Clinical Trial Center, Chonbuk National University Hospital, Jeonju, South Korea Urology, Samsung Medical Center, "Biomedical Research Institute

Purpose: Prostate-specific antigen (PSA) is the most important marker in the diagnosis and follow-up of patients with prostate cancer. The primary objective of this study was to evaluate the effect of various urologic procedures in prostatic area on serum free and total PSA levels. Subjects and methods: A series of 62 patients ( 8 after digital rectal examination [DRE], 12 after transrectal ultrasonography [TRUS], 11 after rigid cystoscopy, 13 after prostatic massage, 8 after TRUS-guided prostate biopsy, and 10 after transurethral resection of prostate [TURP]) were enrolled in the study. Blood samples were taken from each patient before procedure and at 10, 30, 60, and 120 minutes after procedures.

Results: Prostate massage, rigid cystoscopy, TURP, and TRUS-guided prostate biopsy caused statistically significant rise in total and free PSA levels in the serum. There was no significant increase in total and free PSA levels in the serum after DRE and TRUS. The mean differences were greater for free PSA level in the serum for TURP, TRUS-guided prostate biopsy, prostate massage, and rigid cystoscopy.

Conclusion: Total and free PSA levels in the serum are altered by prostate massage, rigid cystoscopy, TRUS-guided prostate biopsy, and TURP. The PSA rises were related to the stimulation strength of the procedures. The total and free PSA levels were increased significantly from 10 minutes after procedures, except DRE and TRUS, and were increased to maximal level at 60 minutes after procedures.

Keywords: prostate specific antigen (PSA), marker, diagnosis, prostate cancer, urologic procedures

\section{Introduction}

\section{What's known?}

- Serum prostate specific antigen (PSA) has become the most useful marker for diagnosis, staging, monitoring, and predicting prognosis of prostate cancer.

- Serum PSA level may be elevated in patients with prostate cancer, benign prostate hyperplasia (BPH), infection, abscess or infarction, transurethral resection of prostate.

\section{What's new?}

- The PSA rises were related to the stimulation strength of the procedures.

Department of Urology, Medical School, Chonbuk National University, Jeonju,

$561-712$, South Korea

Tel +82632501510

Fax $+8263250 I 564$

Email rain@chonbuk.ac.kr
- After prostate massage, rigid cystoscopy, transrectal ultrasono guided prostate biopsy and transurethral resection of prostate, the total and free PSA levels were increased significantly from $10 \mathrm{~min}$ after procedures and increased to maximal level at $60 \mathrm{~min}$ after procedures. 


\section{What is the factor to affect prostate- specific antigen?}

Prostate-specific antigen (PSA) is a kallikrein-like serine protease produced exclusively by prostatic epithelial cells. Serum PSA has become the most useful marker for diagnosis, staging, monitoring, and predicting prognosis of prostate cancer. Current screening recommendation by the American Urological Association and the American Cancer Society includes yearly serum PSA determination in low-risk groups, starting at 50 years of age. High-risk men are encouraged to obtain yearly PSA measurements, starting at 40 years of age. ${ }^{1,2}$ Elevated serum PSA levels are probably a product of disruption of cellular architecture within the prostatic gland. The loss of the barrier afforded by the basal layer and basement membranes within the normal gland is a likely site for the egress of PSA into the circulation. Serum PSA level may be elevated in patients with prostate cancer, ${ }^{3,4}$ benign prostate hyperplasia (BPH), ${ }^{5,6}$ infection, abscess, or infarction. ${ }^{7,8}$ Transurethral resection of prostate (TURP), ${ }^{9}$ transrectal ultrasonography (TRUS)-guided prostate biopsy, ${ }^{10,11}$ ejaculation, ${ }^{12}$ cystoscopy, ${ }^{10}$ colonoscopy, ${ }^{13}$ and coronary artery stent implantation $^{14}$ can cause serum PSA rises. Serum PSA levels can decrease after prostatectomy, treatment with $5 \alpha$-reductase inhibitor, luteinizing hormone-releasing hormone analog and antiandrogen, and radiation. ${ }^{15-19}$

For the patients with lower urinary tract symptoms (BPH/ LUTS), serum PSA, DRE and TRUS were evaluated in the same urologic clinic. The cystoscopy or expressed prostatic secretion by prostatic massage was added to routine practice for evaluation of BPH/LUTS when patients had hematuria or pelvic pain. These procedures seem to affect the cutoff level of serum PSA for decision of prostate biopsy, especially between 2.5 and $10 \mathrm{ng} / \mathrm{mL}$ of serum PSA. Thus, we conducted a prospective study of serum PSA levels consecutively in the same patients undergoing procedures in the prostatic area within 120 minutes of the procedure.

\section{Patients and methods}

We conducted a prospective study on the effect of various urologic procedures in prostatic area for the change of total and free PSA levels in the serum. After Chonbuk National University Hospital Institutional Review Board's approval, 62 men were enrolled in the study. All patients provided informed consent after a candid discussion. We excluded patients who had prostatic biopsy, cystoscopy, or urinary tract infection in the preceding 30 days. The half-life of total serum PSA has been reported to be between 2.2 and 3.2 days, ${ }^{20,21}$ thus, 30 days might be enough time to return to normal range of PSA after prostatic biopsy, cystoscopy, or urinary tract infection. We also excluded patients with a history of prostate cancer, prostatic surgery, luteinizing hormone-releasing hormone analog or antiandrogen use, and $5 \alpha$-reductase inhibitor use.

The patients underwent TRUS, DRE, and prostate massage for the evaluation of their BPH/LUTS. The patients underwent rigid cystoscopy for the evaluation of microscopic or gross hematuria. The patients underwent TRUS-guided prostate biopsy because of abnormal DRE or serum PSA levels over $4.0 \mathrm{ng} / \mathrm{mL}$. All DREs were performed by same physician, with the patient in the lateral position. All prostate massages were performed by same physician. TRUS was performed with a 7.5 MHz transducer (B\&K 2102, Bruel and Kjær, Nærum, Denmark), and 12 core biopsies were taken in all cases with an 18G Tru-cut needle (Pro-MagTM biopsy needle, Medical device technologies, Inc., Gainesville, FL, USA). Rigid cystoscopies were performed with a 20-French cystoscope after lubricating the urethra with $10 \mathrm{~mL}$ of lidocaine gel in our outpatient department. The TURP was performed using a 17-French resectoscope as an inpatient procedure.

Venipuncture was performed, and $5 \mathrm{~mL}$ of blood was collected immediately before procedures. Venipuncture was performed by a urologic resident, and it was maintained with a plastic cannula (Venflon, Viggo, Helsingborg, Sweden). The blood samples were collected again at 10, 30,60, and 120 minutes. The serum was immediately centrifuged and was kept frozen at $-80^{\circ} \mathrm{C}$ until assay. Total and free PSA were measured using an immunoradiometric assay (CIS bio international, Gif-Sur-Yvette Cedex, France).

For multiple comparisons in a small number of patients, we used the Wilcoxon signed-rank test. The Wilcoxon signed-rank test is a nonparametric statistical hypothesis test used when comparing two related samples, matched samples, or repeated measurements on a single sample to assess whether their population mean ranks differ. The mean total and free PSA levels in the serum at baseline were compared with the mean total and free PSA levels in the serum at 10 minutes. In the same way, the mean total and free PSA levels in the serum at baseline were compared with the mean total and free PSA levels in the serum at 30, 60, and 120 minutes, respectively. The results were considered significant at $P<0.05$. All statistical analyses were performed using SPSS version 13.0 (SPSS, Chicago, IL, USA).

\section{Results}

A total of $8,12,13,11,8$, and 10 patients who underwent DRE, TRUS, prostatic massage, rigid cystoscopy, 
Table I Baseline characteristics of patients enrolled in the study

\begin{tabular}{llllll}
\hline Procedures & Number of patients & Age (years) & Prostate volume $(\mathbf{m L})$ & Total PSA $(\mathbf{n g} / \mathbf{m L})$ & Free PSA $(\mathbf{n g} / \mathbf{m L})$ \\
\hline DRE & 8 & $60.34 \pm I 1.31$ & $23.95 \pm 15.28$ & $1.39 \pm 1.12$ & $0.29 \pm 1.23$ \\
TRUS & 12 & $57.02 \pm 12.45$ & $25.33 \pm 18.29$ & $1.51 \pm 1.35$ & $0.23 \pm 1.63$ \\
Prostate massage & 13 & $58.8 \pm 14.78$ & $32.86 \pm 17.55$ & $3.89 \pm 4.48$ & $0.48 \pm 1.56$ \\
Rigid cystoscopy & 11 & $66.14 \pm 15.26$ & $31.35 \pm 22.35$ & $0.28 \pm 1.35$ & $0.06 \pm 0.53$ \\
Prostate biopsy & 8 & $72.93 \pm 14.91$ & $46.1 \pm 21.95$ & $23.15 \pm 18.75$ & $1.89 \pm 1.63$ \\
TURP & 10 & $71.45 \pm 16.28$ & $52.7 \pm 18.48$ & $7.74 \pm 3.35$ & $2.05 \pm 1.42$ \\
\hline
\end{tabular}

Note: Data are expressed as mean \pm standard deviation.

Abbreviations: PSA, prostate-specific antigen; min, minutes; DRE, digital rectal examination; TRUS, transrectal ultrasonography; TURP, transurethral resection of prostate.

TRUS-guided prostate biopsy, and TURP were enrolled this study, respectively (Table 1). The mean age was 63.95 years ( $35-83$ years). The mean ages were $60.34 \pm 11.31$, $57.02 \pm 12.45,58.8 \pm 14.78,66.14 \pm 15.26,72.93 \pm 14.91$, and $71.45 \pm 16.28$ years in patients who underwent DRE, TRUS, prostate massage, rigid cystoscopy, TRUS-guided prostate biopsy, and TURP, respectively (Table 1). The mean prostate volumes were $23.95 \pm 15.28,25.33 \pm 18.29,32.86 \pm 17.55$, $31.35 \pm 22.35,46.1 \pm 21.95$, and $52.7 \pm 18.48 \mathrm{~mL}$ in patients who underwent DRE, TRUS, prostate massage, rigid cystoscopy, TRUS-guided prostate biopsy, and TURP in TRUS measurement, respectively (Table 1).

After prostate massage, rigid cystoscopy, and TURP, the total and free PSA levels increased significantly from 10 minutes after procedures $(P<0.01$; Tables 2 and 3 , Figure 1$)$. And TRUS-guided prostate biopsy also caused the total and free PSA to rise significantly from 10 minutes after procedure $(P<0.05$; Tables 2 and 3, Figure 1). The total and free PSA levels increased to a maximal level at 60 minutes after procedures (Tables 2 and 3, Figure 1). Interestingly, the mean changes were greater for free PSA than total PSA rises $(P<0.05)$. This was most apparent following TURP and TRUS-guided prostate biopsy. But there was no statistically significant change in total and free PSA levels in the serum before and after DRE and TRUS.

\section{Discussion}

PSA has become the most useful marker for diagnosis, staging, monitoring, and predicting prognosis of prostate cancer. However, some forms of urologic procedure increase PSA level. ${ }^{9,10,20,22}$ These unpredictable factors usually require the urologist to repeat previously performed PSA tests. Thus, it is important to know what kind of urologic procedure is affecting PSA level. In our study, after prostate massage, rigid cystoscopy, TRUS-guided prostate biopsy, and TURP, the total and free PSA levels were increased significantly from 10 minutes after procedures and increased to a maximal level at 60 minutes after procedures. The PSA rises were related to the stimulation strength of the procedures.

First of all, DRE is an important examination for screening prostate cancer together with PSA. Long et $\mathrm{al}^{9}$ demonstrated $^{-}$ that serum total and complexed PSA, which were statistically significant, increased at 20 minutes after DRE in 34 patients. Ornstein et $\mathrm{al}^{20}$ also showed that a DRE caused a modest rise in total PSA level in the serum. But, Lynn et al, ${ }^{11}$ showed that there was no significant increase in total PSA level in the serum levels after DRE. The results of our study are similar to the existing findings in that no significant increase in serum total and free PSA at 10, 30, 60, and 120 minutes after DRE. These results suggested that DRE did not disrupt cellular architecture within the prostate gland.

Table 2 The mean changes in total PSA level in the serum before and after procedures according to time

\begin{tabular}{|c|c|c|c|c|c|}
\hline Procedures & Before & After 10 minutes & After 30 minutes & After 60 minutes & After 120 minutes \\
\hline DRE $(n=8)$ & $1.39 \pm 1.12$ & $1.58 \pm 1.33$ & $\mathrm{I} .59 \pm \mathrm{I} .37$ & $1.63 \pm 1.26$ & $\mathrm{I} .6 \mathrm{I} \pm \mathrm{I} .4 \mathrm{I}$ \\
\hline TRUS $(n=12)$ & $\mathrm{I} .5 \mathrm{I} \pm \mathrm{I} .35$ & $1.65 \pm 1.29$ & $1.66 \pm 1.22$ & $1.68 \pm 1.05$ & $1.5 \mathrm{I} \pm \mathrm{I} .18$ \\
\hline Prostate massage $(n=13)$ & $3.89 \pm 4.48$ & $6.68 \pm 9.31 * *$ & $6.81 \pm 5.76 * *$ & $6.72 \pm 6.24 * *$ & $6.58 \pm 5.83 * *$ \\
\hline Rigid cystoscopy $(n=I \mid)$ & $0.28 \pm 1.35$ & $0.59 \pm 2.41 * *$ & $0.62 \pm 2.03 * *$ & $0.7 \mathrm{I} \pm \mathrm{I} .49 * *$ & $0.52 \pm 1.45 * *$ \\
\hline Prostate biopsy $(n=8)$ & $23.15 \pm 18.75$ & $40.10 \pm 27.83^{*}$ & $38.07 \pm 31.2 I^{*}$ & $44.72 \pm 24.9 I^{*}$ & $38.09 \pm 17.52 *$ \\
\hline TURP $(n=10)$ & $7.74 \pm 3.35$ & $|5.34 \pm 4.5|^{* *}$ & $25.87 \pm 8.13^{* *}$ & $29.18 \pm 7.25 * *$ & $22.7 I \pm 5.67 * *$ \\
\hline
\end{tabular}

Notes: $* P<0.05$ versus control by Wilcoxon signed-rank test. $* * P<0.01$ versus control by Wilcoxon signed-rank test.

Abbreviations: PSA, prostate-specific antigen; min, minutes; DRE, digital rectal examination; TRUS, transrectal ultrasonography; TURP, transurethral resection of prostate. 
Table 3 The mean changes in free PSA level in the serum before and after procedures according to time

\begin{tabular}{|c|c|c|c|c|c|}
\hline Procedures & Before & After 10 minutes & After $\mathbf{3 0}$ minutes & After 60 minutes & After I 20 minutes \\
\hline DRE $(n=8)$ & $0.29 \pm 1.23$ & $0.4 I \pm 2.12$ & $0.41 \pm 1.76$ & $0.43 \pm 1.35$ & $0.42 \pm 1.71$ \\
\hline TRUS $(n=12)$ & $0.23 \pm 1.63$ & $0.26 \pm 1.85$ & $0.27 \pm 2.54$ & $0.27 \pm 1.94$ & $0.26 \pm 1.39$ \\
\hline Prostate massage $(n=13)$ & $0.48 \pm 1.56$ & $2.02 \pm 1.33 * *$ & $2.21 \pm 2.13 * *$ & $2.04 \pm 1.87 * *$ & $1.82 \pm 1.23 * *$ \\
\hline Rigid cystoscopy $(n=\mid I)$ & $0.06 \pm 0.53$ & $0.23 \pm 1.14^{* *}$ & $0.24 \pm 1.28 * *$ & $0.21 \pm 1.35 * *$ & $0.18 \pm 0.94 * *$ \\
\hline Prostate biopsy $(n=8)$ & $1.89 \pm 1.63$ & $|5.87 \pm 7.5|^{*}$ & $13.66 \pm 8.62^{*}$ & $12.63 \pm 10.46 *$ & $|3.05 \pm||.9| *$ \\
\hline TURP $(n=10)$ & $2.05 \pm 1.42$ & $6.64 \pm 4.29 * *$ & $13.03 \pm 12.11 * *$ & $15.02 \pm 9.73^{* *}$ & $10.45 \pm 7.47^{* *}$ \\
\hline
\end{tabular}

Notes: ${ }^{*} P<0.05$ versus control by Wilcoxon signed-rank test. ${ }^{*} * P<0.01$ versus control by Wilcoxon signed-rank test.

Abbreviations: PSA, prostate-specific antigen; min, minutes; DRE, digital rectal examination; TRUS, transrectal ultrasonography; TURP, transurethral resection of prostate.
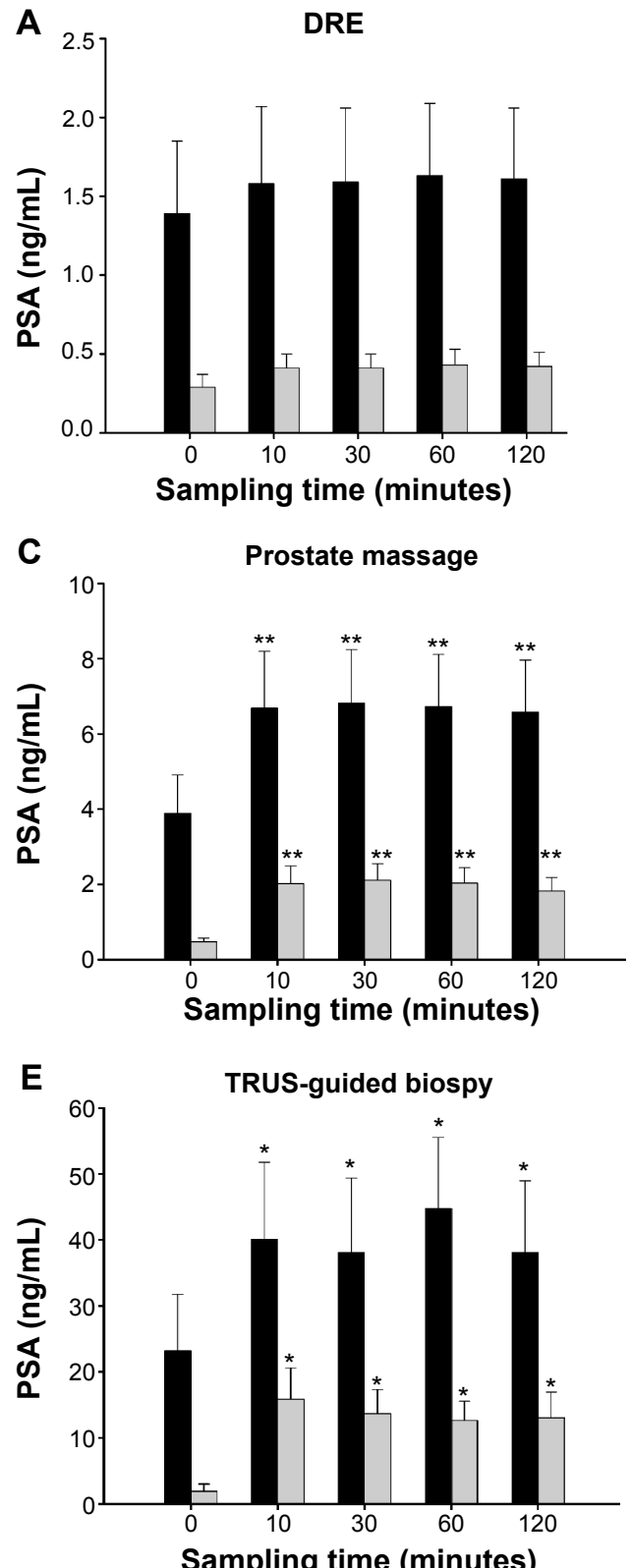
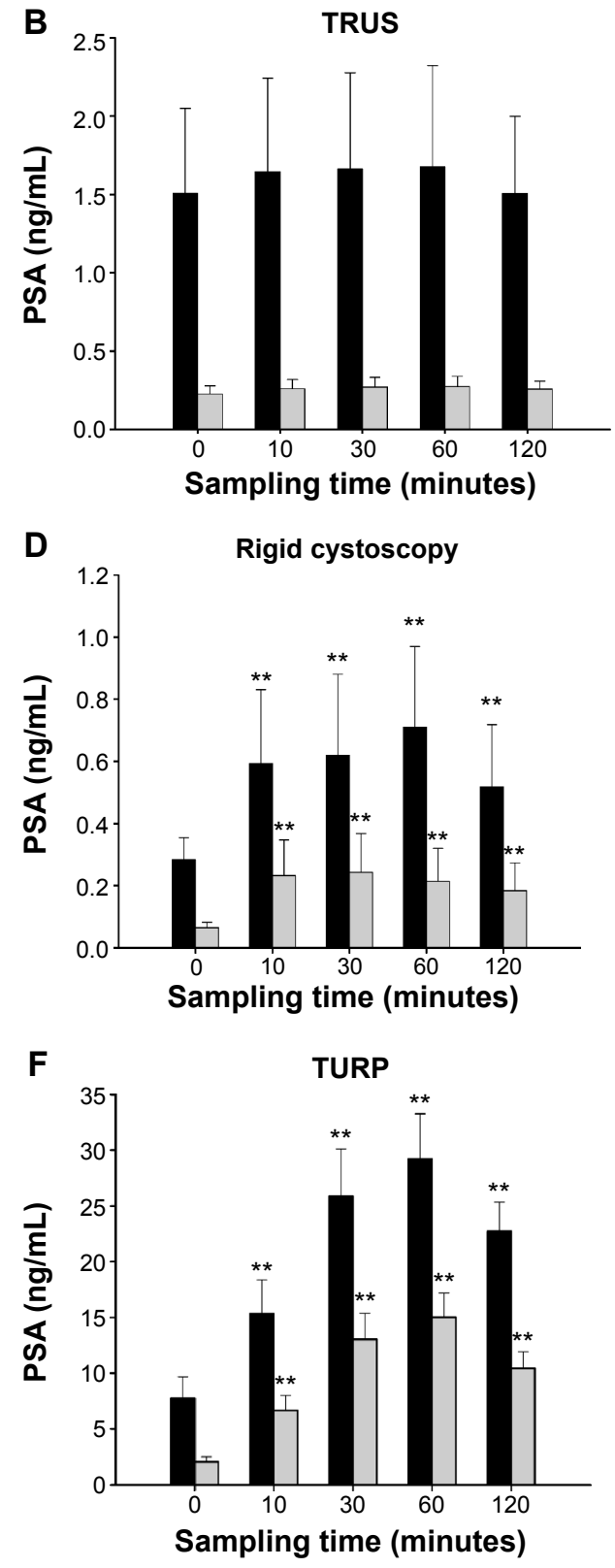

$\square$ TPSA $\square$ FPSA

Figure I The changes in total and free PSA levels in the serum before and after procedures.

Notes: (A) The median PSA level after DRE, (B) TRUS, (C) prostate massage, (D) rigid cystoscopy, (E) prostate biopsy, and (F) TURP. $* P<0.05$ by Wilcoxon signed-rank test. $* * P<0.0$ I by Wilcoxon signed-rank test.

Abbreviations: DRE, digital rectal examination; PSA, prostate-specific antigen; TRUS, transrectal ultrasonography; TURP, transurethral resection of the prostate; TPSA, total prostate-specific antigen; FPSA, free prostate-specific antigen. 
Urethrocystoscopy is the standard procedure for endoscopic evaluation of the lower urinary tract. Collins et $\mathrm{al}^{10}$ noted a change in the mean total PSA level in the serum level of $2.48-2.50 \mathrm{ng} / \mathrm{mL}(P=0.841)$ and in free PSA level in the serum of $0.40-0.49 \mathrm{ng} / \mathrm{mL}(P=0.019)$ in 26 patients at 30 minutes after flexible cystoscopy. Long et $\mathrm{al}^{9}$ showed changes in the mean total PSA level in the serum of $0.68 \mathrm{ng} / \mathrm{mL}(P=0.0006)$ in 28 patients at 20 minutes after flexible cystoscopy and $0.13 \mathrm{ng} / \mathrm{mL}(P=0.0199)$ in 17 patients at 20 minutes after rigid cystoscopy. DeCastro et a ${ }^{22}$ found a small increase in the total and free PSA levels in the serum after flexible cystoscopy. They suggested that although statistically significant, the increase in mean total PSA level in the serum of $0.113 \mathrm{ng} / \mathrm{mL}$ was not clinically relevant. They concluded that flexible cystoscopy does not adversely affect the serum PSA values in men with total serum PSA values $<4 \mathrm{ng} / \mathrm{mL}$. Lynn et $\mathrm{al}^{11}$ also showed that there was no significant increase in serum PSA level after flexible cystoscopy. In our study, the mean changes in total PSA level in the serum levels were $0.31,0.34,0.43$, and $0.24 \mathrm{ng} / \mathrm{mL}$ at $10,30,60$, and 120 minutes after procedures, respectively $(P<0.01)$. No volunteer had a total PSA $>2.5 \mathrm{ng} / \mathrm{mL}$ on the initial serum PSA measurement. One volunteer had a total PSA level in the serum increase from $0.564 \mathrm{ng} / \mathrm{mL}$ before cystoscopy to $2.84 \mathrm{ng} / \mathrm{mL}$ at 10 minutes after cystoscopy and $3.11 \mathrm{ng} / \mathrm{mL}$ at 30 minutes after cystoscopy. Although the effect of cystoscopy on serum PSA was clinically minimal, serum PSA should be checked before performing cystoscopy.

Prostatic massage and DRE are commonly performed at the same urologic visit, as is serum PSA measurement. Several studies have investigated the effect of prostatic massage on total PSA level in the serum levels, with different conclusions. Tarhan et $\mathrm{al}^{23}$ showed that the total and free PSA levels in the serum increased significantly after prostate massage and that no significant increase occurred in the mean total PSA level in the serum after prostatic massage in patients with prostate cancer. In our study, the total and free PSA levels in the serum significantly increased after prostatic massage, and the rise in free PSA level in the serum after prostatic massage was greater.

TRUS is a useful tool for evaluation of prostate volume and architecture as well as for prostate biopsy. Long et $\mathrm{al}^{9}$ and Lynn et $\mathrm{al}^{11}$ suggested that TURP and TRUS-guided prostate biopsy significantly increased total PSA level in the serum. And they demonstrated that serum complexed PSA is less prone to variations compared to total PSA level in the serum. In our study, TRUS alone had no effect on total and free PSA levels in the serum. But TRUS guided prostate biopsy and TURP had significant effect on total and free PSA levels in the serum. The change of free PSA level in the serum was greater than total PSA level in the serum. The results of our study are consistent with results of Long et $\mathrm{al}^{9}$ and Lynn et al. ${ }^{11}$ Elevated serum PSAs are probably a product of disruption of cellular architecture within the prostate gland. This can occur in the setting of prostate disease (prostate cancer, prostatitis) and with prostatic manipulation (TURP, prostatic massage, prostate biopsy). Although TURP and TRUS-guided prostate biopsy increased serum total and free PSA levels, it is unlikely to be of clinical relevance as these changes in level were minimal.

A limitation of our study is a very small cohort of patients for each urological procedure. However, to our knowledge, this study represents the first prospective investigation of the change of total and free PSA levels in the serum at baseline, $10,30,60$, and 120 minutes after each procedure. It is clinically important because most urologic procedures are taken within 120 minute for evaluation. This study gives valuable information about change of PSA after various urological procedures as time passes.

\section{Conclusion}

This study found increased total and free PSA levels in the serum after prostatic massage, rigid cystoscopy, TRUS-guided prostate biopsy, and TURP. But DRE and TRUS had no effect on total and free PSA levels in the serum. Prostate manipulation has minimal effect on total and free PSA when patients visit urologic clinic for their BPH/LUTS. But, serum PSA measurement must precede prostatic massage and rigid cystoscopy when prostate massage and rigid cystoscopy are added to routine checkup.

\section{Acknowledgments}

This study was supported by grants of the Korea Healthcare technology R\&D Project, Ministry of Health, Welfare and Family Affairs, Republic of Korea (HI13C0104).

\section{Author contributions}

Seung Chol Park, Yu Seob Shin, Dal Sik Kim, Sung Zoo Kim, Nam Cheol Park, and Jong Kwan Park were involved in the laboratory procedures, data collection, and analysis. Seung Chol Park, Yu Seob Shin, Nam Cheol Park, and Jong Kwan Park were involved in the preparation and the review of the article. All authors contributed toward data analysis, drafting and revising the paper and agree to be accountable for all aspects of the work.

\section{Disclosure}

The authors report no conflicts of interest in this work. 


\section{References}

1. American Urological Association. Early Detection of Prostate Cancer: Policy Statement. Board of Directors Minutes. Linthicum, MD: American Urological Association; 1992.

2. Mettlin C, Jones G, Averette H, Gusberb SB, Murphy GP. Defining and updating the American Cancer Society guidelines for the cancerrelated checkup of prostate and endometrial cancers. CA Cancer J Clin. 1993;43:42-46.

3. Catalona WJ, Smith DS, Ratliff TL, et al. Management of prostatespecific antigen in serum as a screening test for prostate cancer. $N$ Engl J Med. 1991;324:1156-1161.

4. Crawford ED, DeAntoni EP, Etzieri R, Schaefer VC, Olson RM, Ross CA. Serum prostate-specific antigen and digital rectal examination for early detection of prostate cancer in a national community-based program. Urology. 1996;47:863-869.

5. Mochtar CA, Kiemeney LA, van Riemsdijk MM, et al. Prostatespecific antigen as an estimator of prostate volume in the management of patients with symptomatic benign prostate hyperplasia. Eur Urol. 2003;44:695-700.

6. Yu DW, Park SC, Seo IY, Rim JS. Can serum PSA predict prostate volume in men with benign prostate hyperplasia? Korean J Urol. 2005;46:574-578.

7. Agnihotri S, Mittal RD, Kapoor R, Mandhani A. Asymptomatic prostatic inflammation in men with clinical BPH and erectile dysfunction affects the positive predictive value of prostate-specific antigen. Urol Oncol. 2014;32:946-951.

8. Tchetgen MB, Oesterling JE. The effect of prostatitis, urinary retention, ejaculation and ambulation on the serum prostate-specific antigen concentration. Urol Clin N Am. 1997;24:283-291.

9. Long R, Giri S, Diver S, Duddy L, McKeown D, Moran K. Effect of prostate manipulation on the serum levels of complexed prostatespecific antigen and total prostate-specific antigen. Int $J$ Urol. 2006;13:947-950.

10. Collins GN, Martin PJ, Wynn-Davies A, Brooman PJ, O’Reilly PH. The effect of digital rectal examination, flexible cystoscopy and prostatic biopsy on free and total prostate specific antigen, and the free-to total prostate specific antigen ratio in clinical practice. $J$ Urol. 1997;157:1744-1747.

11. Lynn NNK, Collins GN, O’Reilly PH. Prostatic manipulation has a minimal effect on complexed prostate-specific antigen levels. BJU Int. 2000;86:65-67.
12. Lim JH, Kim SD, Choi KS, Choi SB, Kim DW, Park JK. The changes of serum prostate-specific antigen after ejaculation. Korean $J$ Urol. 2007:48:1247-1250.

13. Schwartz BF, Faught J, Jezior JR. The effect of colonoscopy on serum prostate antigen levels. BJU Int. 1999;84:302-304.

14. OzcanT, Bozlu M, Muslu N, Gozukard KH, Seyis S, Akcay B. Elevation of the serum total and free prostate specific antigen levels after stent implantation in patients with coronary artery disease. Swiss Med Wkly. 2009; 139:672-675.

15. Lange PH, Ercole CJ. The value of serum prostate specific antigen determinations before and after radical prostatectomy. J Urol. 1989;141:873-879.

16. Stamey TA, Kabalin JN, Ferrari M. Prostate specific antigen in the diagnosis and treatment of adenocarcinoma of the prostate. III. Radiation treated patients. $J$ Urol. 1989;141:1084-1087.

17. Stamey TA, Kabalin JN, Ferrari M, Yang N. Prostate specific antigen in the diagnosis and treatment of adenocarcinoma of the prostate. IV. Anti-androgen treated patients. J Urol. 1989;141:1088-1090.

18. Morgan WR, Zincke H, Rainwater LM, Myers RP, Klee GG. Prostate specific antigen value after radical prostatectomy for adenocarcinoma of the prostate. Impact of adjuvant treatment (hormonal and radiation). J Urol. 1991;145:319-323.

19. Etzioni RD, Howlader N, Shaw PA, et al. Long-term effects of finasteride on prostate specific antigen level: results from the prostate cancer prevention trial. J Urol. 2005;174:877-881.

20. Ornstein DK, Rao GS, Smith DS, Ratliff TL, Basler JW, Catalona WJ. Effect of digital rectal examination and needle biopsy on serum total and percent of free prostate specific antigen levels. J Urol. 1997;157:195-198.

21. Oesterling JE, Chan DW, Epstein JI, et al. Prostate specific antigen in the preoperative and postoperative evaluation of localized prostatic cancer treated with radical prostatectomy. J Urol. 1988;139:766-772.

22. DeCastro BJ, Baker KC. Effect of flexible cystoscopy on serum prostatespecific antigen values. Urology. 2009;73:237-240.

23. Tarhan F, Orcun A, Kucukercan I, Camursoy N, Kuyumcuoglu U. Effect of prostatic massage on serum prostate-specific antigen levels. Urology. 2005;66:1234-1238.
Clinical Interventions in Aging

\section{Publish your work in this journal}

Clinical Interventions in Aging is an international, peer-reviewed journal focusing on evidence-based reports on the value or lack thereof of treatments intended to prevent or delay the onset of maladaptive correlates of aging in human beings. This journal is indexed on PubMed Central, MedLine,

\section{Dovepress}

CAS, Scopus and the Elsevier Bibliographic databases. The manuscript management system is completely online and includes a very quick and fair peer-review system, which is all easy to use. Visit http://www.dovepress. com/testimonials.php to read real quotes from published authors. 